\title{
Conductive Composites of Lignophenol and Polyaniline
}

\author{
Mitsuru Aoyagi and Masamitsu Funaoka \\ Graduate School of Bioresources, Mie University, Japan Science and Technology Agency (JST), \\ Solution Oriented Research of Science and Technology (SORST) \\ Fax; +81-59-231-9517, e-mail: aoyagi@bio.mie-u.ac.jp
}

Lignophenols (LPs) have been directly synthesized from lignocellulosics through the phase-separation system composed of concentrated acids and phenols under mild conditions. As resulting LPs have rich phenolic hydroxyl groups by grafted phenols, various derivatives were easily designed from LPs. LPs and Polyaniline (PAni) produced conductive composites mixed in homogeneous conditions of $N$-methylpyrollidone solutions with emeraldine salts confirmed by FT-IR and UV-Vis spectroscopy. Softwood LPs grafted with mono phenol showed better conductivities. Especially, both Western Hemlock (Tsuga heterophylla)-lignophenol ( $p$-cresol type) and Western Hemlock-lignophenol ( $m$-cresol type) showed high conductivities, $4.6 \times 10^{-6}$ $\mathrm{Scm}^{-1}$ and $1.0 \times 10^{-7} \mathrm{Scm}^{-1}$, respectively. But both LPs with polyphenols and $2^{\text {nd }}$ derivatives of LPs with small molecules have not showed good results. The conductivities of LPs/PAni composites probably were produced by polymeric structures, hydrophobic $\pi-\pi$ stackings and intermolecular interactions. Thus, these properties of LPs for dopings were derived from both polymeric structures with aromatic rings and high acidities amplified by 1,1-bis(aryl)propane type structures. These conductivities were sufficient for removal of static electricity or resistance for rust.

Key words: lignin, lignophenol, polyaniline, composites

\section{INTRODUCTION}

Lignophenol is lignin-based phenolic polymer which has been directly synthesized from lignocellulosics through the phase-separation system [1]. The polymer with 1,1-bis(aryl)propane-2-O-aryl-3-ol type units (Fig.1) is tried to be applied for various products such as molds, additives, medicals, adhesives and electronics $[2,3]$. These applications were realized by particular properties such as solubility, plasticity, recyclability, and sustainability. Especially composites with other materials were synthesized due to interactions of rich hydroxyl groups and aromatic rings.

Conductive polymers have been attracted as materials for electronics since Shirakawa et al succeeded synthesis of polyacethylene thin film in 1971[4]. After the breakthrough, it was found that conductivity of polyacetylene increased at 9-12 magnitudes by doping of electron donors or acceptors [5]. Consequently polyacethylene showed high conductivity $\left(10^{5} \mathrm{Scm}^{-1}\right)$ equal to cupper in 1987. Other conductive polymers

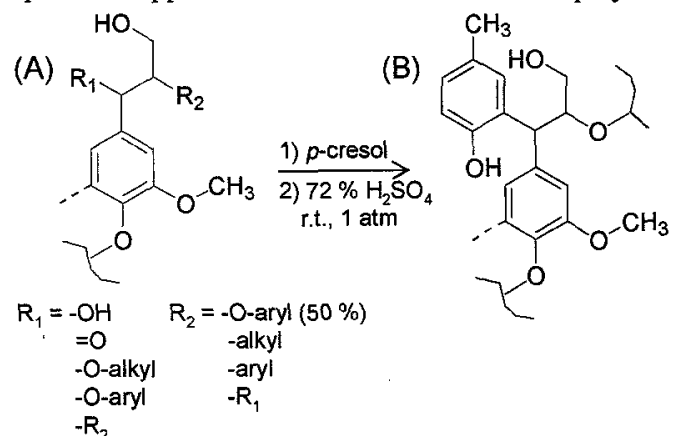

Fig.1 Synthesis of lignophenol ( $p$-cresol type) directly from lignocellulosics. (A) Softwood native lignin in lignocellulosics and (B) lignophenols ( $p$-cresol type)
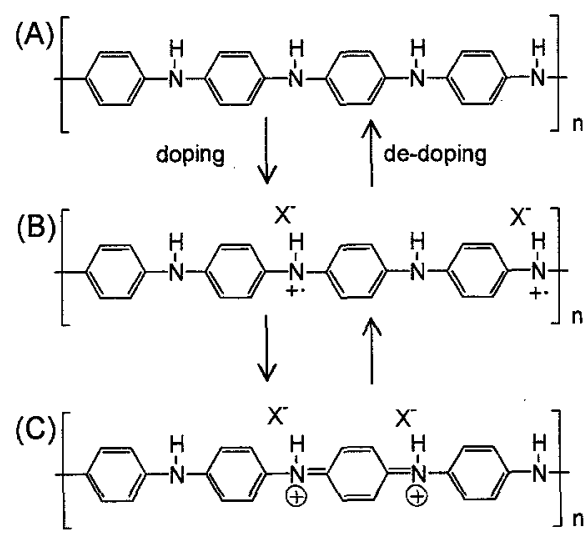

Fig.2 Structures of polyaniline. (A)Emeraldine base, (B)Emeraldine salt (ES) with polaron structure (semi-quinone radical cation) and (c)ES with bipolaron structure

have been also discovered such as polypyrol, polythiphene, poly-p-phenylene, and polyaniline. These compounds were applied for electronics materials. For example, polypyrol and poly- $p$-pheneylene were used as condensers in cellular phones and electroluminescence devices in displays [6], respectively. In this way, conductive polymers innovated in new market of polymer science.

Polyaniline (PAni) was discovered by McDamid from aniline black [7]. PAni also has been attracted because of easy synthesis, low cost, thermal stability and high solubility. In fact, PAni has been applied for cathode materials in market [8]. Although PAni consists of 4 type structures, leucoemeraldine base (yellow), pernigranine base (black), emeraldine base (EB, blue) 
and emeraldine salt (ES, green), only ES shows conductivity (Fig.2) [9]. These structures were changed by reversible redox reactions. Moreover, ES was produced from EB by protonation with reversibility.

Thus, in this study an attempt has been made to compose recyclable bio-based polymer with valuable conductive polymer. Here, polymeric dopings of lignophenols for PAni were tried by mixing in $\mathrm{N}$-methylpyrollidone.

\section{EXPERIMENTAI}

2.1 Synthesis of lignophenols

Western Hemlock (WH, Tsuga heterophylla) was used as a softwood material. The woody material was milled for 80 mesh passed. Extractives were removed by acetone at room temperature for $72 \mathrm{hrs}$. Lignophenols have been synthesized following the phase-separation system, using two different techniques. First, one-step method [1] was carried out for WH. The milled wood of WH $(1.0 \mathrm{~g})$ was solvated by $10 \mathrm{~mL}$ of $p$-cresol for $5 \mathrm{~min}$ with stirring, and then $15 \mathrm{~mL}$ of $72 \% \mathrm{H}_{2} \mathrm{SO}_{4}$ was immersed into the mixture. This heterogeneous mixture was vigorously stirred at $30^{\circ} \mathrm{C}$. After 60 min stirring, the mixtures were separated for two different phases, $p$-cresol layer and $\mathrm{H}_{2} \mathrm{SO}_{4}$ layer, by centrifugation. The $p$-cresol layer was thrown into diethyl ether (EtOEt) under stirring in ice bath to remove $p$-cresol. Resulting precipitation was extracted using acetone for $8 \mathrm{hrs}$ under stirring. This acetone extraction was dropped into EtOEt in ice bath, resulting precipitation after evaporating and drying on $\mathrm{P}_{2} \mathrm{O}_{5}$, lignophenol was obtained.

Second, two-step method (process II, Fig.3) was also carried out for WH as follows [2]. The material $(500 \mathrm{~g})$ was thrown into acetone solution of phenol analogs such as $p$-cresol or $m$-cresol with concentration of $3 \mathrm{~mol} /$ phenylpropane units $\left(\mathrm{C}_{9}\right.$ units), which were subunits of native lignin. After evaporation of acetone, $72 \% \mathrm{H}_{2} \mathrm{SO}_{4}$ aq. solutions were immersed into the materials adsorbed by $p$-cresol at $30{ }^{\circ} \mathrm{C}$. Then the mixtures were stirred vigorously soon after mixing. After $60 \mathrm{~min}$, the mixtures were thrown into $20 \mathrm{~L}$ of de-ionized water with vigorously stirred with a homogenizer for $5 \mathrm{~min}$. Then the purple precipitations were washed until $\mathrm{pH}=5$.

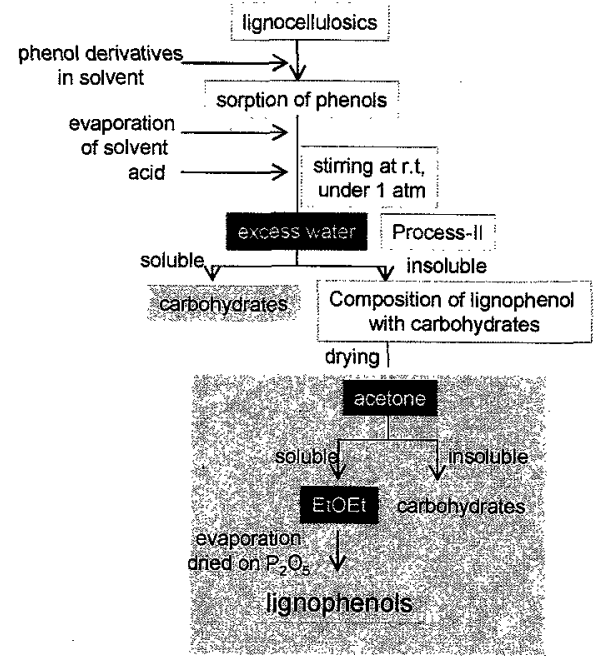

Fig.3 Synthesis flow of lignophenols through the phase-separation system, twostep method.
After drying the precipitates, lignophenols were extracted by acetone. The lignophenols in acetone were purified by thrown into diethyl ether (EtOEt) under vigorously stirring in chilled conditions. After evaporating and drying on $\mathrm{P}_{2} \mathrm{O}_{5}$, lignophenol were obtained.

\subsection{Characterization of lignophenols}

The structure of lignophenol was characterized by Gel Permeation Chromatography (GPC), ${ }^{1} \mathrm{H}-\mathrm{NMR}$ and Thermal Mechanical Analysis (TMA). GPC was carried out by LC-10 system (Shimadzu Co.) with four columns (KF801, KF802, KF803 and KF804, Shodex Co.), using tetrahydrofuran (THF) after distillation as eluent. $M_{w}$ and $M_{n}$ were determined based on standard polystylene. ${ }^{1} \mathrm{H}$-NMR spectrum was measured by JNM-A500 (JOEL Co.) in $\mathrm{CDCl}_{3}$ or $\mathrm{CDCl}_{3} / \mathrm{C}_{5} \mathrm{D}_{5} \mathrm{~N}=3 / 1(\mathrm{v} / \mathrm{v})$. TMA was also carried out by TMA-SS (SII Inc.) in the temperature range $50-280{ }^{\circ} \mathrm{C}$ at a rate of $2{ }^{\circ} \mathrm{Cmin}^{-1}$, using penetrating technique for a measurement. UV-Vis spectroscopy was carried out on a UV-560 (JASCO Co.) with integral sphere apparatus. FT-IR spectroscopy was also carried out on a Spectrum GX (Perkin Elmer Co.), using the $\mathrm{KBr}$ pellet technique for sample preparation.

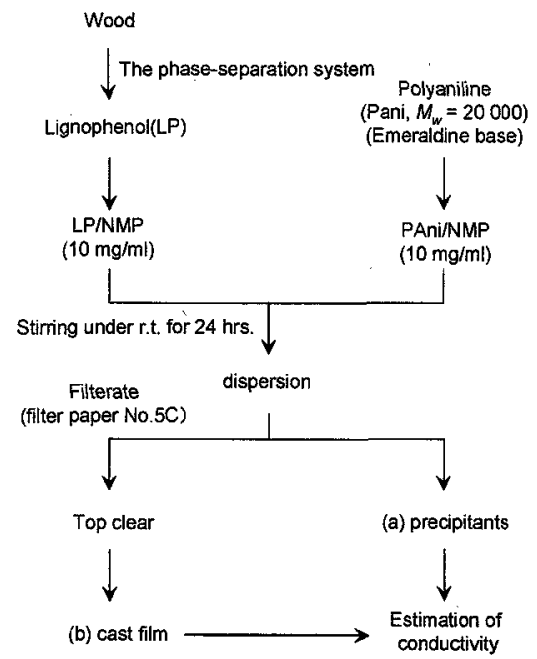

Fig.4 Procedure for mixing of lignophenols/PAni in N-methylpyrollidone solutions.

2.3 Preparation of lignophenol / polyaniline composites

Lignophenol / polyaniline composites (LP/PAni) have been prepared by mixing of each $N$-methylpyrollidone (NMP) solution (Fig.4). After mixing these two solutions, the mixtures were stirring for $48 \mathrm{hrs}$ at room temperature. Resulting precipitations were collected on 5C filter papers $(40 \mathrm{~mm} \phi)$ by Kiriyama funnels. The precipitates were dropped on slide grasses at $60^{\circ} \mathrm{C}$. The resulting LP/PAni films were obtained by evaporation. Conductivities of both precipitations and films were estimated by Potentionstat (HA-105, Hokuto Denko Co.).

\section{RESULT AND DISCUSSION}

3.1 Conductivity of lignophenol-polyaniline composites LP/PAni showed conductivities $10^{-6} \mathrm{Scm}^{-1}$ which are equal to semiconductors in spite of weak acidity of lignophenol. As shown in Fig.5, Western 
Hemlock-lignophenol ( $p$-cresol type) synthesized by one-step method (WHLC1) showed the best conductivity, $3.86 \times 10^{-6} \mathrm{Scm}^{-1}$. Other lignophenols also showed relatively high conductivity. For example, $90 \mathrm{wt} \%$ of WHLC synthesized by two-step method (WHLC2) and $75 \%$ of Western Hemlock-lignophenol ( $m$-cresol type) synthesized by two-step method

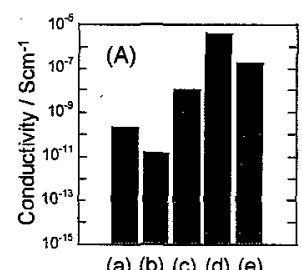

(a) (b) (c) (d) (e)

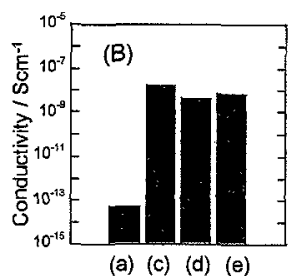

(a) (c) (d) (e)
Fig.5 Electric conductivities of $(\mathrm{A})$ aggregated precipitants on the $5 \mathrm{C}$ filter papers and (B) cast film. (a) Emeraldine base of PAni, (b) filter paper, (c)lignosulfoic acid, (d) Western Hemilock(Tsuga heterophy/la)-lignophenol(p-cresol type, WHLC) synthesized by phaseseparation system, 1 step method/PAni $=75 / 25$ (w/W) and (e) WHLC by 2 step method PAni $=90 / 10(\mathrm{w} / \mathrm{w})$.

(WHLmC2) with PAni had $1.78 \times 10^{-7} \mathrm{Scm}^{-1}$ and $6.80 \times$ $10^{-8} \mathrm{Scm}^{-1}$, respectively. Because PAni (EB) and WHLC1 showed $10^{-12} \mathrm{Scm}^{-1}$ and $10^{-11} \mathrm{Scm}^{-1}$, respectively, the conductivities were produced by doping of LP. It is sufficient for LP/PAni to apply such as electrics removal materials. Generally, lignophenols grafted with monophenols acted as good dopants.

Futhermore, lignophenols grafted with polyphenols such as catechol, resorcinol and pyrogallol showed lower conductivities. Because of high frequency of hydrogen bonds based on rich hydroxyl groups, these results implied contribution for doping of hydrogen bonding is lower than the $\pi-\pi$ interaction. These results indicated that hydrophobic $\pi-\pi$ interactions were needed for lignophenol doping.

\subsection{Characteristics of lignophenols}

To investigate a mechanism of doping properties of LP to PAni, characterization of LP was achieved. Fig.6 illustrates diffused and reflected UV-Vis spectrum for WHLC1/PAni on filter paper. Two distinguished absorption peaks and one broad band were observed at $\lambda$ $=430,800$ and $600 \mathrm{~nm}$, respectively. The former two peaks were estimated as bipolaron of emeraldine salts [10] shown in Fig.6. The absorption of 430 and $800 \mathrm{~nm}$ were observed owing to polaron-bipolaron transition [11] and the $\pi-\pi$ transition of localized polaron, respectively

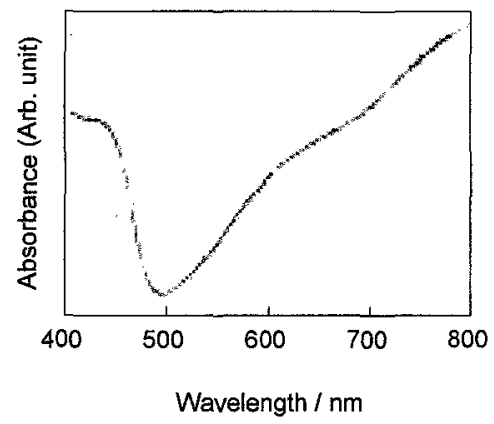

Fig.6 The visible light absorbance spectrum for cast film of WHLC1/PAni.
[12]. The latter broad band was due to the $\pi-\pi$ transition of the exinton absorption of the quinoid type structure in PAni (EB), which was reduced form with a dark blue appearance [13]. This result indicated incomplete protonation of the imine structures in the LP/PAni composites [14]. Therefore, conductivity of LP/PAni can be developed by improvement for the protonation.

Fig.7 demonstrates FT-IR spectra for WHLC1/PAni. The peaks between $2000-1700 \mathrm{~cm}^{-1}$ demonstrated quinoid type structures on PAni shown in Fig.2 (B) and (C). These results indicated that emeraldine salts, which were conductive structures for PAni, were produced by only mixing in NMP solutions. Moreover absorption of $\mathrm{N}-\mathrm{H}$ stretching mode observed at $3500 \mathrm{~cm}^{-1}$ for PAni based on free imine structures. The notable shifts were due to significant occurrence of hydrogen bonding between PAni and LP[10]. In fact, $\mathrm{O}-\mathrm{H}$ stretching mode with hydrogen bonds of LP disappeared. But free $\mathrm{N}-\mathrm{H}$ stretching was also observed in LP/PAni because of undoped PAni (EB) observed by UV-Vis spectroscopy.

When the two solutions were mixed, insoluble moieties often produced by aggregation. The aggregated precipitates had higher conductivities than the cast films of precipitates. This result also implied that polymeric structures tended to compose with conductive structures of PAni.

\subsection{Mechanisms}

As concerned with weakness of acidity for phenols, the good performances of LP/PAni can be explained in two ways. First, increase of acidity by amplified phenolic hydroxyl groups on 1,1-bis(aryl)propane type structures, which exist frequently in the molecules, promoted doping for PAni. The phenolic hydroxyl groups of WHLCl were stabilized by core units of lignin linking $\mathrm{Cl}$. Because there are few carboxyl acid and carbonyl groups in lignophenol (Fig.7), acidic doping with protonation was carried out only phenols. In fact, $\mathrm{pKa}$ of phenolic hydroxyl groups in dimethylformamide (DMF) decreased to 12.3 compared to phenol ( $\mathrm{pKa}=19.8$ in DMF) measured by cyclic voltanmetry using Pt electrodes [15].

Second, increase of interactions between LP and PAni also promoted electron mobility (Fig.8). Not only

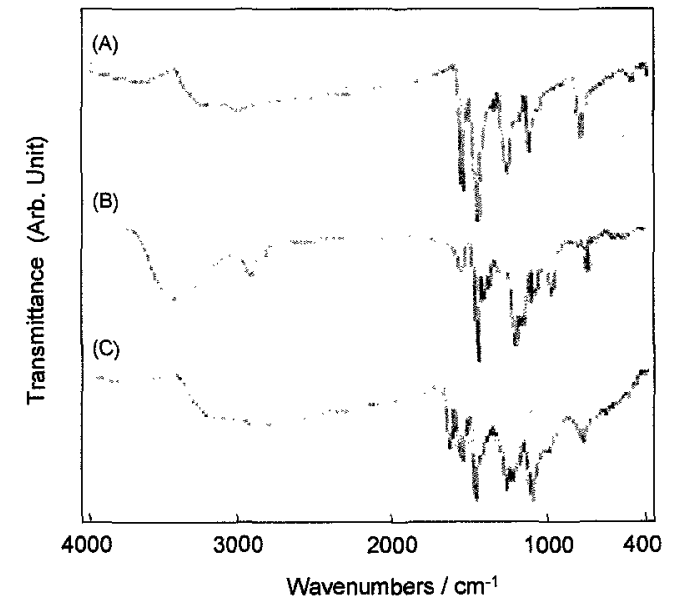

Fig.7 FT-IR spectrum for (A) PAni (EB), (B) WHLC2 and (C)WHLC2/ PAni (75/25) on the filter paper. 
hydroxyl groups, but rich p-electrons probably concerned with intermolecular interactions like $\pi-\pi$
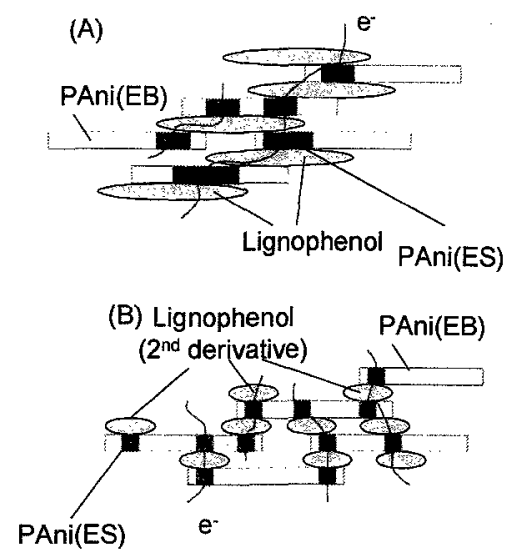

Fig.8 Schematic models for conductivities in lignophenolpolyaniline(LP-PAni) composites. (A) Doping with polymeric lignophenol, (B)doping with $2^{\text {nd }}$ derivatives of lignophenol. Line showed conductive routes by PAni (emeraldine salts, ES) produced by lignophenols and polyaniline (emeraldine base, EB).

stackings. Because lignophenol second derivatives $[2,3]$ with small molecules $\left(M_{w}=1500-1000\right.$, GPC), derived under alkaline condition, with high acidity in DMF (pKa $=11.5)$ have showed relatively low conductivity $\left(10^{-11}\right.$ $\mathrm{Scm}^{-1}$ ). This result implied doping by LP needed polymeric structures. In addition, no influences of other acidic moieties, such as sulfuric acid and carboxyl groups, were estimated, because there were not both $\mathrm{SO}_{4}{ }^{2-}$ or acidic groups in WHLC confirmed by $\mathrm{BaSO}_{4}$ or FT-IR, respectively.

Therefore lignophenol doping for PAni was occurred by both phenolic hydroxyl groups and $\pi-\pi$ stacking of aromatic rings.

Consequently, these conductivities were sufficient for removal of static electricity or resistance for rust. Because optimizations are expected by both modifications for lignophenols and methods for mixing, various applications such as electronics devices mainly composed of biomass can be realized.

\section{ACKNOWLEDGEMENT}

The authors thank to the supports of Japan Science and Technology Agency (JST), Solution Oriented Research on Science and Technology (SORST).

\section{REFERENCES}

[1] M. Funaoka and I. Abe, Tappi., 72(8), 145-149 (1989).

[2]M. Funaoka, Polym. Int., 47, 277-290(1998)

[3]M. Funaoka, Macromol. Symp., 201, 213-221(2003)

[4]H. Shirakawa, S. Ikeda, Polymer Journal, 2, 231-244(1971)

[5]H. Shirakawa, E. J. Louis, A. G. McDiamid, C. K. Chang, A. J. Heeger, Journal of Chemical Society, Chemical Communication, 578-580(1977)

[6]R. H. Friend, D. D. C. Bradley, P. D. Townsend, Journal of Physics D : Applied Physics, 20, 1367-1384(1987)

[7]A. G. McDarmid, J. C. Chang, M. Halpern, W. S.
Huang, S. L. Mu, N. L. D. Somasiri, W. Wu, S. I. Yaniger, Mol. Cryst. Liq. Cryst., 121, 173-180(1985)

[8]J. Anand, S. Palaniappn, D. N. Sathyarayana, Prog. Polym. Sci., 23, 993-1018(1998)

[9]N. Kuramoto, Shikizai Kyokaishi, 76(9), 352-359(2003)

[10]P. Ghosh, A. Chakrabarti, S. K. Siddhanta, Eur. Polym. J., 35, 803-813(1999)

[11]R. Gangopadhyay, A. De, G. Ghosh, Synth: Met., 123, 21-31(2001)

[12]Y. Kang, S. K. Kim, C. Lee, Mater. Sci. Eng. C, 24, 39-41(2004)

[13]M. S. Cho, S. Y. Park, J. Y. Hwang, H. J. Choi, Mat. Sci. Eng. C, 24, 15-18(2004)

[14]H. S. moon, J. K. Park, Synth. Met., 92, 223-228(1998)

[15]W. C. J. Barrette, H. W. J. Johnson, D. T. Sawer, Anal. Chem., 56, 1890-1898(1984)

(Received February 16. 2007;Accepted July 12, 2007) 